\title{
Multiple endocrine neoplasia type 2B
}

INSERM

\section{Source}

INSERM. (1999). Orphanet: an online rare disease and orphan drug data base. Multiple endocrine neoplasia type 2B. ORPHA:247709

Multiple endocrine neoplasia 2B (MEN2B) syndrome is a rare aggressive form of MEN2 (see this term) characterized by medullary thyroid carcinoma (MTC, see this term), pheochromocytoma (see this term), mucosal gang lioneuroma, and marfanoid habitus. 